\title{
A multi-scale finite element analysis and sectional design approach for the creep of polymeric FRC
}

\author{
Rutger Vrijdaghs \& Lucie Vandewalle \\ Department of Civil Engineering, KU Leuven, Belgium \\ M. di Prisco \\ Department of Structural Engineering, Politecnico di Milano, Italy
}

ABSTRACT: This paper presents the experimental, numerical and analytical results of a multi-scale investigation into the uniaxial tensile creep behavior of polymeric fiber reinforced concrete (FRC). In an extensive experimental program, the short-term and creep behavior of individual fibers, the fiber-matrix interface and the composite material are investigated. The short-term and creep properties are used to calibrate the material models of a finite element model with discrete fibers, which allows to determine the creep of polymeric FRC under tensile loading. A Monte-Carlo analysis is performed to assess the influence of the fiber dispersion and sustained load level on the time-dependent crack widening. Finally, the numerical results are used in a sectional approach that allows to translate the uniaxial tensile creep behavior into a flexural creep prediction. The proposed methodology can be readily implemented into design codes, to allow for the creep deformation of cracked FRC to be taken into account.

\section{INTRODUCTION}

Since the introduction of fiber reinforced concrete (FRC) in the Model Code 2010 (MC2010) (fib 2010), designers can use this composite material in structural applications (di Prisco 2009). While design rules are given to take the post-cracking tensile capacity of the material into consideration, long-term performance due to creep is not accounted for. Creep of FRC under tension is of high importance in structural design and the subject has been gaining attention in recent years (Boshoff \& Nieuwoudt 2017, Buratti \& Mazzotti 2017).

In order to fully understand the mechanisms behind the creep of cracked FRC, a multi-scale experimental program has been completed. In total, over 270 tests (both under short-term and creep loading up to 9 months) were completed on the fiber, fiber-matrix interface and FRC scale. The results of the multiscale program were used to calibrate and validate a two-phased finite element model in which a uniaxial tensile creep load is applied to a specimen. The numerical results were then used in a sectional approach that allowed for stress redistribution and time-dependent crack width growth under sustained flexural loading.

\section{EXPERIMENTAL WORK}

The multi-scale experimental program considers short-term characterization and sustained uniaxial tensile loading tests on three distinct scales: (1) fiber (2) fiber-matrix interface and (3) composite, i.e. FRC, scale.

\subsection{Fiber behavior}

This paper reports one type of polypropylene (PP) fiber which is commercially available in Belgium. The fiber (named B) is characterized as macro-synthetic fiber and are validated to be used in structural applications in accordance with EN 14899-2. The diameter and length is $0.7 \mathrm{~mm}$ and $55 \mathrm{~mm}$ respectively. Shortterm characterization tests are performed according to the newly revised standard EN 14889 and the mechanical properties are reported in Table 1 , with $f_{t}, \varepsilon_{f t}$ and $E$ the tensile strength, the strain at maximum stress and the cord modulus respectively. The coefficient of variation is shown in brackets.

Table 1: Mechanical properties of the fibers

\begin{tabular}{llll}
\hline & $f_{t}[\mathrm{MPa}]$ & $\varepsilon_{f t}[\%]$ & $E[\mathrm{MPa}]$ \\
\hline B & $490(0.8 \%)$ & $9.5 \%(2.0 \%)$ & $6300(3.5 \%)$ \\
\hline
\end{tabular}


In addition to short-term testing, creep tests on 6 specimens were completed at load ratios (LRs) between $22 \%$ and $63 \%$ of $f_{t}$. Some specimens were kept under load for up to 270 days. The creep deformations are shown in Figure 1. More details about individual fiber behavior can be found in literature (Vrijdaghs 2017a).

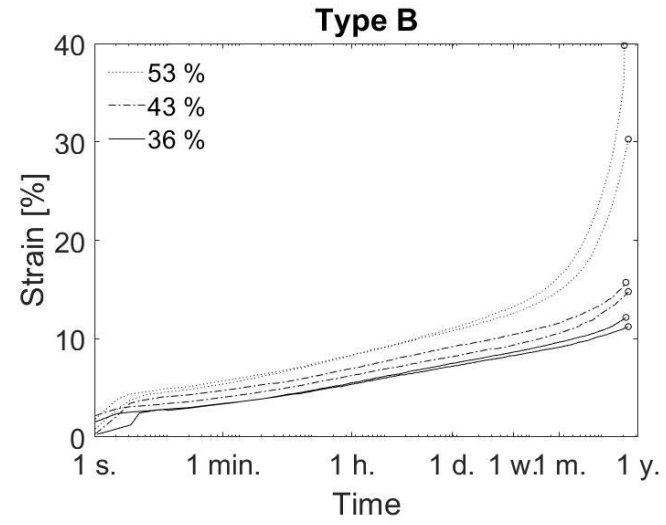

Figure 1. Single fiber creep deformations

\subsection{Pull-out behavior}

The behavior of the composite material is determined by the strength of the bond between fiber and matrix. In order to assess this bond strength, pull-out tests are performed for various values of the embedded length $(10,15$ and $20 \mathrm{~mm})$ and angle $\left(0,15\right.$ and $\left.30^{\circ}\right)$. Additionally, pull-out creep tests are completed for LRs ranging from $25 \%$ to $60 \%$ of the short-term strength as measured on companion specimens at the same embedded length and angle.

All pull-out tests considered pull-out from a concrete matrix, rather than a mortar phase in order to capture as accurately as possible the real behavior in the composite material.

The short-term pull-out results for an embedded angle of $15^{\circ}$ are shown in Figure 2, where it is noted that the pull-out response can be divided into 3 parts: (1) force build-up (2) instantaneous loss of strength following the maximum force (3) oscillating load pattern or fiber fracture. This has been observed for other combination of the angle and length as well.

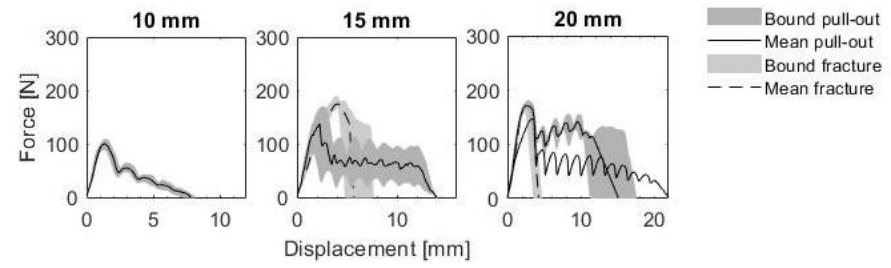

Figure 2. Pull-out response at various embedded lengths

The pull-out creep deformations are measured under controlled environmental conditions and a sustained load is kept on the specimen for up to 270 days. The results of single fiber pull-out creep is shown in Figure 3.

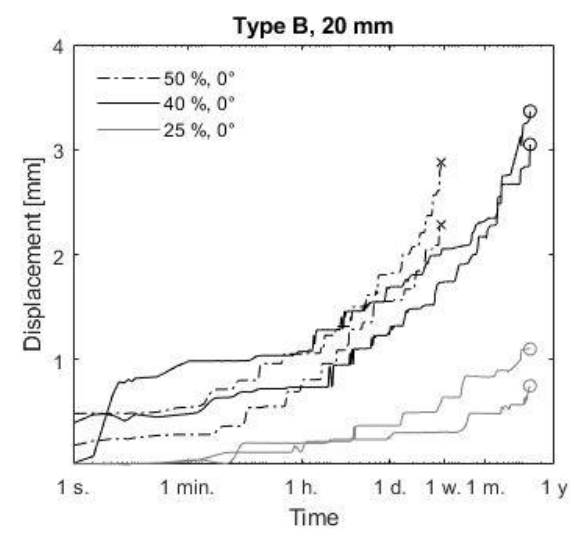

Figure 3. Single fiber pull-out creep

It has been shown that single fiber creep deformations are the driving force behind pull-out creep deformations and a positive feedback loop mechanism is identified in which time-dependent Poisson contractions cause further pull-out creep deformations. Further details on the pull-out tests setup and identification of the pull-out mechanisms is discussed elsewhere (Vrijdaghs 2017b).

\subsection{FRC behavior}

The composite behavior is identified under various types of loading. In all FRC tests, fibers are added to account for $1 \mathrm{~V} \%$ and all tests are performed after 28 days of curing. The characterization of FRC is standardized in Europe in accordance with EN 14651, where a notched three point bending test is used to measure the post-cracking residual tensile strength. The results of the characterization test is shown in Figure 4.

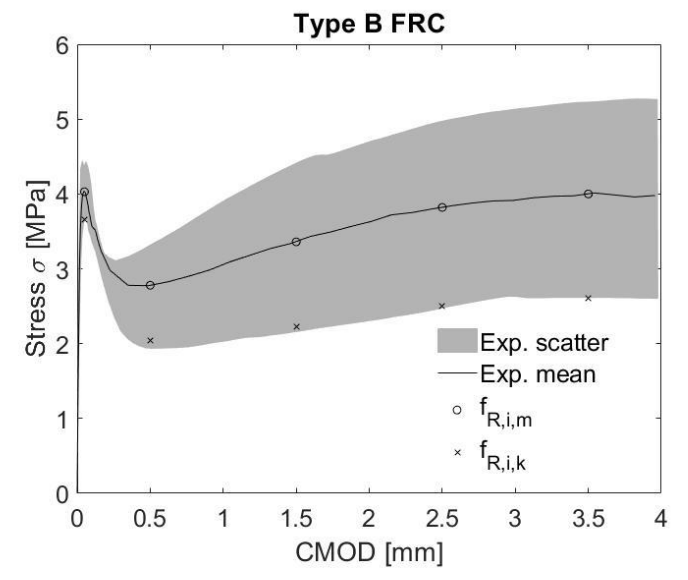

Figure 4. Bending characterization according to EN 14651

Using the classification as outlined in MC10, the post-cracking strength class is $2 \mathrm{~d}$. Simultaneously, the compressive strength class is identified as C30/37, representing a normal strength concrete.

The notched bending tests yields two similarly sized halves of the beam. From each half, a concrete core is taken with a diameter and height of $100 \mathrm{~mm}$ and $300 \mathrm{~mm}$, respectively. At half height, these concrete cores are notched to reduce the diameter to $80 \mathrm{~mm}$. Subsequently, the notched cores are 
precracked to an initial crack width of $0.20 \mathrm{~mm}$ in the notched section. The precracking procedure is done manually and allows for a stable uniform crack width growth during the test, independent of the fiber distribution in the cracked section. The precracked cores are then subjected to sustained uniaxial tensile loading amounting to $30 \%$ or $45 \%$ of the residual strength at the precrack width. Each specimen is placed in a specifically designed cantilever based creep frame in a climate chamber at $20{ }^{\circ} \mathrm{C}$ and $60 \%$ humidity. The time-dependent crack widening is measured for 270 days on 14 specimens ( 7 per LR).
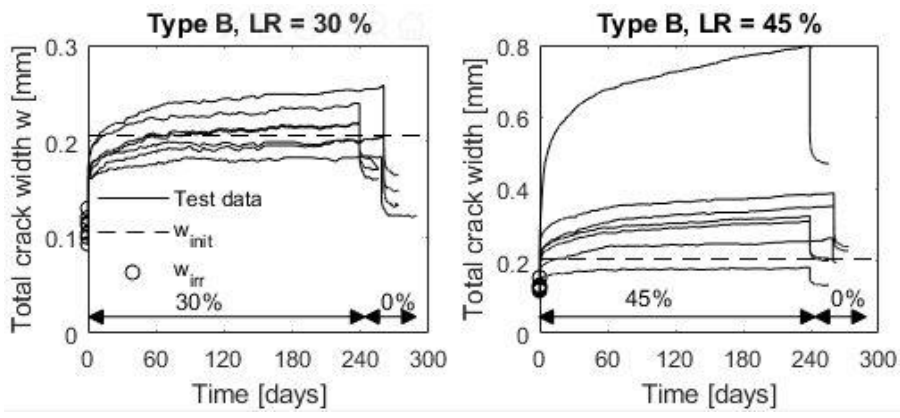

Figure 5. Creep deformations of FRC

The creep deformations are shown in Figure 5.

Further details and experimental results can be in literature (Vrijdaghs 2018).

\section{NUMERICAL MODELING}

While the experimental program yields important results, the high time-, space- and cost constraints of experiments limits the practical study to time frames up to 1 (or several) years. In practice, structures need to satisfy structural requirements during the entire life time of the element. Therefore, numerical modeling can be used to assess the creep deformations for time scales up to 50 years.

In order to capture the structural interaction between fiber and matrix, both in space and in time, a two-phased numerical model is developed and built where fibers are considered separately from the concrete matrix. While this significantly increases the computational cost (especially in contrast with a homogeneous approach to the material), the model can be used to determine the tensile stresses in the fibers crossing the crack. Additionally, the short-term and creep results obtained in the multi-scale experimental program can be used to calibrate the material models of the fibers and matrix, and the resulting numerical crack width growth can be compared against the measured crack widths during the FRC creep tests.

The numerical modeling consists of three phases: (1) preprocessing, i.e. generation of the fibers in the predefined volume, (2) construction of the FEM and simulating the creep deformations and (3) post-processing and comparison with experimental data. In the present approach, the influence of the fiber dispersion is stochastically taken into account by generating 25 different fiber sets. Each fiber sets contains a number of fibers which are randomly distributed throughout the predefined volume. As such, a Monte-Carlo analysis allows to determine the influence of the embedded angle or length on the fiber stress. Furthermore, such an approach can be easily extended to include a preferential fiber orientation and its effect on the time-dependent crack width growth. However, the latter mechanism is not included in this paper.

\subsection{Fiber generation algorithm}

A fiber generation algorithm (FGA) is developed in MATLAB that can generate straight fibers in a user-defined volume, in this case a prism of $150 \times 150 \times 400 \mathrm{~mm}^{3}$, corresponding to one half of the EN 14651 beam. Following input of the required fiber fraction, and the geometrical properties of the fiber, the algorithm consecutively generates new fibers. Each fiber is defined by its two end points, connected by a straight line with a length equal to the fiber length.

The FGA randomly picks a point, representing one end of the fiber, within the defined volume. The orientation of the fiber is defined by two angles $\alpha$ and $\beta$ : the former is the angle of the fiber projection in the xy-plane with the y-axis, and the latter is the angle between the xy-plane and the fiber axis. The location of each fiber is checked to ensure that (1) the end points are within the concrete volume and (2) the fiber is sufficiently far from all other up-until-then generated fibers. Mathematically, the minimum distance between the axis of any two fibers needs to be at least two times the fiber diameter. If both requirements are fulfilled, the fiber location is fixed and a new fiber is generated.

Then, a numerical core with a diameter of $100 \mathrm{~mm}$ is extracted from the beam and is sawn to a height of $300 \mathrm{~mm}$. Both coring and rectifying have an effect on the fibers in the prism, as fibers can be (1) completely within the core, (2) intersecting the core or (3) completely outside the core. In the first case, the entire fiber is kept intact, while the fiber is cut in the second case, retaining only the part within the cylinder. In the third case, the fiber is discarded. Next, the core is notched at mid-height to decrease the diameter to $80 \sim \mathrm{mm}$. Again, fibers can be (partly) cut by the procedure, and the algorithm automatically retains and/or discards the relevant parts for each fiber. Additionally, a crack is introduced in the notched section: two perfectly flat crack faces are separated by an uniform initial crack width, corresponding to the average experimentally observed irreversible crack width after unloading. After the coring, rectifying and notching, the location of all fibers in one fiber set is known. The 
outlined procedure is repeated 25 times in order to assess the influence of the fiber dispersion on the predicted creep results.

\subsection{Construction of the FEM and simulation}

The model consists of a solid concrete mesh in which the fibers are randomly distributed. The FEM is constructed in DIANA which allows the fibers to be defined independently from the concrete mesh. As such, the background concrete mesh remains identical between the different fiber sets and represents the two halves of the notched core.

The FEM consists in total of 4 different element types: (1) 3D solid elements, (2) embedded reinforcements, (3) bond-slip reinforcement elements and (4) beam elements. All element types use quadratic interpolation functions. The 3D solid elements are tennode, three-side isoparametric solid tetrahedrons and are used to represent the concrete matrix. The embedded reinforcement elements are used to define fibers that are not crossing the crack. These fibers contribute to the stiffness of the material, but since they are not crossing any crack, they take up only very limited forces and are therefore assumed to be perfectly bonded. The third element type is a bond-slip reinforcement to model fibers crossing the crack. As these fibers take up significant parts of the tensile load, they cannot be assumed to be perfectly bonded. Therefore, a interfacial bond-slip behavior is defined for every fiber crossing the crack. The fibers can then slide with respect to the concrete matrix at load application. Finally, beam elements are used to model the fibers in the cracked section itself. In the crack, no mother elements are present in which reinforcement element could be embedded. Therefore, bond-slip reinforcement elements are connected by beam elements in the crack.

The pull-out displacements of the bond-slip fibers are defined and calibrated based on the short-term pull-out test data. Since every bond-slip fiber has a different embedded angle and length, each fiber is assigned a unique material model. The creep of the fibers is taken into account by implementing a time-dependent stiffness material model for the beam elements in the crack. Since the experimental program on the fiber-matrix interface identified the single fiber creep deformations as the driving force behind pull-out creep, the time-dependent pull-out deformations are lumped in the single fiber creep curves implemented for the beam elements. As such, pull-out creep is (only) implicitly taken into account.

The boundary conditions of the numerical model are simple and are only imposed on two nodes. On the bottom of the concrete core, a node is placed directly on the axis of the core, and this node is fixed in all three translational directions. Conversely, on the top on the concrete core, a node on the core's axis is fixed in the horizontal directions, but is free to deform in the vertical direction. Note that the solid concrete element only has translational DOFs so rotational boundary conditions cannot be assigned.

The load is applied to the specimen at the nodal location of the top boundary condition. At this point, a tensile load is applied along the core's axis equal to $30 \%$ or $45 \%$ of the average experimental residual strength at the precrack width of $0.2 \sim \mathrm{mm}$. The load is applied instantaneously at $\mathrm{t}=1 \mathrm{~s}$ and the total simulated time period is 50 years, divided into 225 logarithmically spaced time steps.

Finally, to ensure stability in the non-linear analysis, a linear stiffness iterative scheme is implemented where the stiffness matrix is computed only once every time step. In order to speed up as much as possible the iteration scheme, the first iteration in each time step is a simple scalar multiplication of the previous incremental solution through the implementation of a continuation algorithm. Fiber fracture, and the associated required stress redistribution, are numerically controlled through the use of the line search algorithm. For every incremental time step, convergence needs to be reached within at most 500 iterations. Convergence is reached if the displacement and force norm (i.e. the ratio of the Euclidian norm after the current iteration to the Euclidian norm of the initial unbalance) are simultaneously satisfied with a tolerance value equal to $2 \%$.

\subsection{Numerical results and comparison}

All simulations are ran on a 6-core, 12-threated Xeon processor with 32 GB RAM on a Windows 10 platform. The total calculation time consists of both the fiber generation time as well as the simulation in DIANA. For both fiber types, the complete generation and simulation of 1 fiber sets takes around 8.5 hours, with both parts lasting roughly equally long. The entire process is automated so that all 25 fiber sets can be simulated without manual intervention.

In Figure 6, the average experimental creep deformations are shown for every creep specimen at a load ratio of $30 \%$ (left) and $45 \%$ (right). Additionally, the average numerical predictions across all 25 fiber sets is shown, together with a scatter band representing $80 \%$ of all specimens. Finally, the average, minimum and maximum creep deformation predicted by the numerical model are also shown.
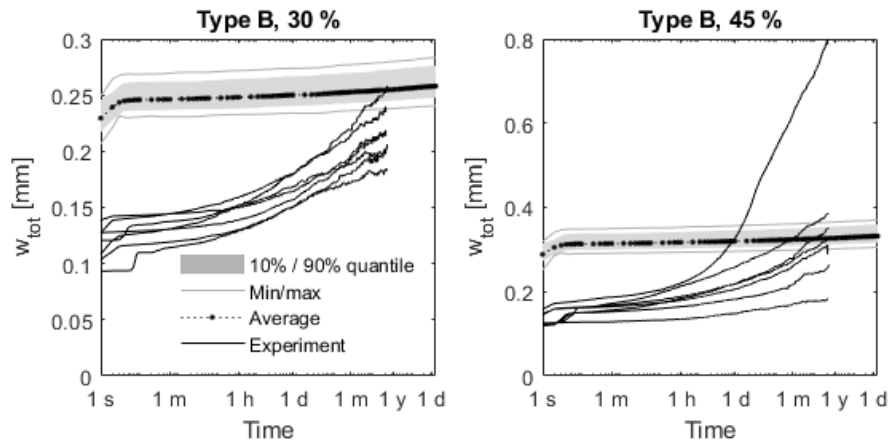

Figure 6. Comparison of experimental and FEM data 
The comparison between the experimental and numerically obtained creep curves indicate that the model is able to capture the crack width growth to an acceptable degree, given the various assumptions. The instantaneous and first-week creep deformations are overestimated by the model, owing to assumption of a perfectly flat crack surface. Indeed, upon nonuniform crack opening, the crack partially closes, which in turn is counteracted by the protruding aggregates in the experiments. However, in the numerical model, no such retardation mechanism exists and fibers are free to deform without any obstruction.

The numerical results show that no structural failure is observed, even for time scales up to 50 years.

\section{SECTIONAL APPROACH}

While the numerical analyses can be used to predict the creep deformations after several decades under sustained uniaxial tension loading, in practice, uniaxial tension is of little significance. Indeed, the creep deformations under flexural loading are much more of interest for a designer. Therefore, a sectional approach is presented that can be used to predict timedependent crack width growth for cracked FRC under sustained bending loads. The sectional approach considers the cracked section of the notched EN 14651 beam test and divides the section in a number of elements over the height of the section. Horizontal and bending moment equations can be formulated and expressed as a function of the stress profile over the height of the section.

\subsection{Overview and methodology}

The sectional approach simplifies the constitutive relations in order to keep the computational time in check. Specifically, concrete is assumed to behave linear elastically in compression and a linear-plastic stress-strain relation is assumed in tension. Given then a certain initial precrack width, the stress profile under the assumption of the simplified constitutive relations can be computed such that the horizontal and moment equations are satisfied. Then, the creep curves are assigned to each element in the sectional approach, where the compressive creep model proposed in MC10 is assigned to elements subjected to compression. For the elements in the cracked part of the section, the results of the numerical model are used to predict the creep deformation in each element as a function of its own stress level and time.

For a flexural creep test, the applied bending moment remains constant but the resulting crack width continuously increases due to fiber creep, fiber pullout or fiber rupture. The latter mechanism not only causes an instantaneous increase in the crack width, but requires a stress redistribution in the cracked section as well. Indeed, part of the section cannot take up any forces any more, and the resulting change in stress in all other parts of the section causes a corresponding change in deformation. The sectional analysis can take into account the stress redistribution caused by localized fiber fracture. The resulting changes in deformation for any element can creep in time themselves.

Globally, deformation profile $\delta(t, y)$ as a function of the height in the cracked section $y$ can thus be expressed as the summation over all instantaneous deformations $\Delta \delta_{k}$ (resulting from a change in applied bending moment, or from a stress redistribution following fiber fracture) multiplied by their own timeshifted creep coefficient $\varphi_{k}\left(t, y, t_{k}\right)$. This is expressed in Equation 1.

$$
\delta(t, y)=\sum \phi_{k}\left(t, y, t_{k}\right) \cdot \Delta \delta_{k}\left(t_{k}, y\right)
$$

\subsection{Results and discussion}

Since neither the experimental data, nor the numerical results indicated structural failure for any specimen, the sectional approach never needs to account for stress redistribution. Nevertheless, and in order to highlight the performance of the presented algorithm, it is assumed that a cracked element cannot sustain any further load is the total deformation of that element at any time exceeds a certain limit value. This limit value can be arbitrarily chosen, and in the presented results, it is set to the maximum deformation observed during the precracking procedure of the bending test. Indeed, before the start of the flexural creep simulation, the EN 14651 beam is precracked to a crack mouth opening displacement of $0.5 \mathrm{~mm}$ and the resulting deformation profile over the height is calculated. The maximum observed deformation over the height is assigned to the limit deformation.

In the following, the (simulated) results of a flexural creep tests on 1 specimen, precracked to $0.5 \mathrm{~mm}$ and subjected to a flexural load equal to $50 \%$ of the strength at the precrack level are presented. The mean response is shown as a function of time, together with the minimum and maximum observed response as well as the $10 \%$ and $90 \%$ quantile values.

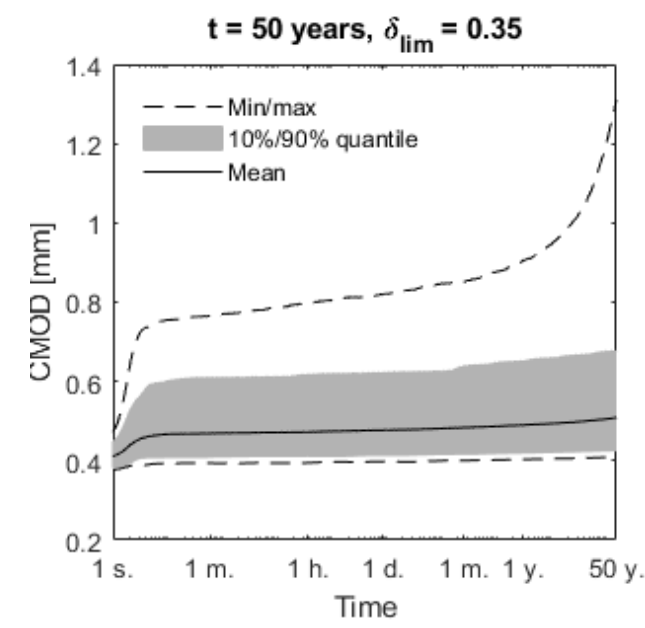

Figure 7. Predicted CMOD in function of time 
In Figure 7, the global response in terms of CMOD as a function of time is shown. While the crack opening remains relatively in check for most predictions (based on the 25 FEM simulations), one particular prediction sees the CMOD increase dramatically, referring to the maximum response. For this particular simulation, a relatively low number of fibers crossed the cracked section in the numerical simulations, leading to a higher creep rate. In the sectional approach, significant stress redistribution is observed which sets in motion a positive feedback loop created by increasing stresses in the remaining part of the section. Indeed, the stress evolution in time for the tensile stress at the bottom of the crack $\sigma_{T}$ and the maximum compressive stress $\sigma_{C}$ is shown to increase, refer to Figure 8.
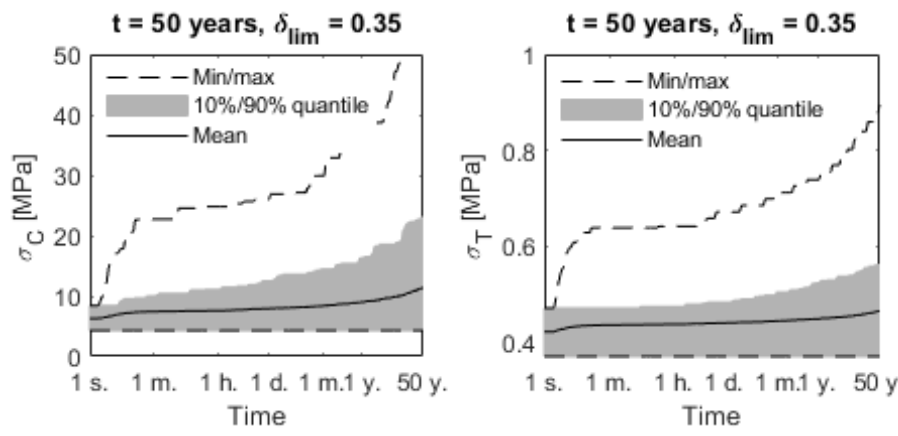

Figure 8. Stress evolution as a function of time

The compressive stress can even exceed the strength of the concrete, and structural failure is predicted for this specimen. It is shown that the presented sectional approach can indeed predict crack widths, stress evolutions and structural failure and can be easily extended or simplified for use in design codes.

\section{CONCLUSIONS}

This paper presents an overview into research on the time-dependent behavior of cracked polypropylene fiber reinforced concrete under sustained uniaxial tensile loading. The research considered a multi-scale experimental program where the short-term properties, as well as the creep behavior is investigated on three different scales: fiber, fiber-matrix interface and composite. The results of the experimental program are used to calibrate and validate a two-phased numerical model, which in turn is used as input for a design-focused sectional approach. The main results of the work are summarized below.

The experimental work has shown that the load ratio greatly influences the behavior across all scales. Higher loads lead to proportionally faster failure and more brittle behavior. Similarly, the fiber behavior was found to be of high importance in the pull-out and composite performance, both under short-term and creep loading.
The two-phased numerical model was shown to be effective to predict the crack width growth upon calibration of the fiber and interface models. Structural failure was not observed even after creeping for 50 years for all simulations in the Monte-Carlo analysis.

A sectional approach is presented that can take into account stress redistribution due to time-dependent fiber fracture or varying load levels. The results show that the global response can be accurately predicted and that the model can predict structural failure. As such, an extension can be made towards inclusion of the approach in design codes.

\section{REFERENCES}

Boshoff WP, Nieuwoudt P. Tensile Creep of Cracked Steel Fibre Reinforced Concrete: Mechanisms on the Single Fibre and at the Macro Level. In: Serna P, Llano-Torre A, Cavalaro SHP, editors. Creep Behaviour in Cracked Sections of Fibre Reinforced Concrete: Proceedings of the International RILEM Workshop FRC-CREEP 2016. Dordrecht: Springer Netherlands; 2017. p. 63-75.

Buratti N, Mazzotti C. Creep Testing Methodologies and Results Interpretation. In: Serna P, Llano-Torre A, Cavalaro SHP, editors. Creep Behaviour in Cracked Sections of Fibre Reinforced Concrete: Proceedings of the International RILEM Workshop FRC-CREEP 2016. Dordrecht: Springer Netherlands; 2017. p. 13-24.

di Prisco M, Plizzari G, Vandewalle L. Fibre reinforced concrete: new design perspectives. Materials and Structures. 2009;42(9):1261-81.

Fédération internationale du béton (fib). Model Code 2010 First complete draft. 2010.

Vrijdaghs R, di Prisco M, Vandewalle L. Creep Deformations of Structural Polymeric Macrofibers. In: Serna P, Llano-Torre A, Cavalaro SHP, editors. Creep Behaviour in Cracked Sections of Fibre Reinforced Concrete: Proceedings of the International RILEM Workshop FRC-CREEP 2016. Dordrecht: Springer Netherlands; 2017a. p. 53-61.

Vrijdaghs R, di Prisco M, Vandewalle L. Short-term and creep pull-out behavior of polypropylene macrofibers at varying embedded lengths and angles from a concrete matrix. Construction and Building Materials. 2017b;147:858-64.

Vrijdaghs R, di Prisco M, Vandewalle L. Uniaxial tensile creep of a cracked polypropylene fiber reinforced concrete. Materials and Structures. 2018;51(1):5. 